\title{
Ovulation after sympathetic denervation of the rat ovary produced by freezing its nerve supply
}

\author{
S. N. Wylie, P. J. Roche and W. R. Gibson \\ Department of Physiology, Monash University, Clayton, Victoria 3168, Australia
}

\begin{abstract}
Summary. The ovaries of cyclic female rats were unilaterally or bilaterally denervated or sham-operated. Denervation was achieved by freezing the ovarian vascular pedicle and suspensory ligament about $1 \mathrm{~cm}$ from the ovary; this technique renders the ovary devoid of innervation for up to 10 days. Denervated ovaries from 6 unilaterally and 7 bilaterally operated animals exhibited normal ovulation rates $(5 \cdot 8 \pm 1 \cdot 0$ and $5 \cdot 2 \pm 1 \cdot 2$ respectively, mean \pm s.e.m.) compared with intact $(5 \cdot 6 \pm 0.4)$ and sham-operated controls ( $4.4 \pm 0.6$ unilaterally sham-operated; $4.1 \pm 0.8$ bilaterally sham-operated). It is concluded that the mechanism of follicular rupture is unaffected by the absence of ovarian sympathetic innervation.
\end{abstract}

\section{Introduction}

It has not been clearly established whether the ovarian sympathetic nerves play any role in the process of ovulation (Bahr, Kao \& Nalbandov, 1974; Mohsin \& Pennefather, 1979). Early studies involving abdominal sympathectomy (Sweet \& Thorp, 1929; Brooks, 1935) suggested that the ovarian sympathetic nerves were not required for ovulation but results from more recent studies seem inconsistent with these findings. For example, the $\alpha$-adrenoceptor blocker, dibenzyline, prevents follicular rupture in rabbits when injected into the aorta just above the ovarian artery (Virutamasen, Hickok \& Wallach, 1971), and in hens when injected systemically or directly into the follicle wall (Ferrando \& Nalbandov, 1969; Kao \& Nalbandov, 1972). Also, local chemical denervation of the rat ovary with 6-hydroxydopamine decreases the numbers of gonadotrophininduced ovulations (Walles, Owman, Sjöberg, Stefenson \& Wallach, 1980).

Other early work examining the effects of denervation on ovulation has not necessarily excluded a role for the ovarian sympathetic nerves. For example, ovulation still occurs after transection or complete removal of areas of the spinal cord (Hinsey \& Markee, 1932). This procedure however, would be expected to leave sympathetic post-ganglionic fibres that could still play a permissive (e.g. trophic) role in follicular function. Also, transplanted ovaries can sometimes ovulate (Friedman, 1929) but often they do not and instead produce unruptured luteinized follicles (Deanesly, 1956; Dominguez \& Riboni, 1971).

In many of these studies, checks were not made to determine whether denervation was complete and that reinnervation had not occurred. This is particularly important if ovulation rate is unaltered because even a few remaining nerves may be enough to maintain normal ovarian function.

The present study was performed to re-examine the effect of ovarian denervation on ovulation rate in the rat. A simple freezing method was used to denervate the ovaries (Gibson, Roche, Summers \& Wylie, 1984) and all ovaries were examined thoroughly for the presence or absence of sympathetic nerves. Part of this work has been reported in abstract form (Wylie, Roche \& Gibson, 1983). 


\section{Materials and Methods}

Animals. Cyclic female Sprague-Dawley rats between 50 and 60 days of age were caged in groups of 4 under controlled conditions of light (lights on 07:00-19:00 h) and temperature $\left(20^{\circ} \mathrm{C}\right)$. Food and water were freely available. Oestrous cycles were monitored before and during the experiment by taking daily vaginal smears. Only those rats exhibiting a regular 4- or 5-day cycle pattern before the experiment were used.

Denervation. Ovaries were denervated by freezing the ovarian vascular pedicle and ovarian suspensory ligament with their accompanying nerves for $20 \pm 5 \mathrm{sec}$, using a metal probe which had been chilled in liquid nitrogen (Gibson et al., 1984). The vessels were frozen immediately proximal to the point where the uterine branch of the ovarian artery originates, about $1 \mathrm{~cm}$ from the ovary. The ovarian suspensory ligament was also frozen about $1 \mathrm{~cm}$ from the ovary. This procedure produces complete adrenergic denervation in $50 \%$ of ovaries judged by the absence of nerves detectable by fluorescence microscopy and of noradrenaline measured by high-performance liquid chromatography (Gibson et al., 1984).

Sham operations were performed using identical procedures except that the probe was not chilled.

Experimental procedure. All operations were performed at metoestrus under general anaesthesia produced by an intraperitoneal injection of a mixture of methohexitone sodium (Brietal Sodium: Eli Lilly, Sydney, Australia), $50 \mathrm{mg} / \mathrm{kg}$, and amobarbital sodium (Amytal Sodium: Eli Lilly, Indianapolis, U.S.A.), $83 \mathrm{mg} / \mathrm{kg}$. Rats were bilaterally denervated (Group B) or shamoperated (Group SB), unilaterally denervated (Group U) or sham-operated (Group SU), or unoperated (Group C). Males were introduced into the cages 3-5 days after surgery so that the time of oestrus in the females could be determined. When spermatozoa were detected in the vaginal smear, females were killed and their ovaries and oviducts removed. All females were killed between 4 and 10 days after surgery, since this is the period in which no adrenergic nerve fibres are found in ovaries successfully denervated by this method (Gibson et al., 1984).

Ovaries from 19 rats in Group U, 14 in Group B and 48 in Groups SU, SB and C were placed in embedding medium (O.C.T. compound, Tissue-Tek, Miles Labs, IL., U.S.A.), frozen in a cryostat and then sectioned at $14 \mu \mathrm{m}$. Five sections from equally spaced regions across each ovary were prepared using fluorescence histochemistry (de la Torre \& Surgeon, 1976). These sections were examined under a fluorescence microscope (Leitz Wetzlar, Germany; $\times 25$ water immersion objective and $\times 10$ eyepiece) to determine the amount of adrenergic innervation present in each. Only those ovaries with no adrenergic nerve fibres in any of their 5 representative sections were considered to be completely denervated.

Oviducts were subjected to routine histological procedures, serially sectioned at $12 \mu \mathrm{m}$ and stained with haematoxylin and eosin. The ovulation rate for each ovary was determined by counting the number of oocytes in the corresponding oviduct.

The ovaries from other rats in Groups $U$ and B were also subjected to routine histological procedures and serially sectioned at $12 \mu \mathrm{m}$. The numbers of fresh corpora lutea (CL) were counted and compared with the corresponding tubal oocyte count. This permitted a check on the accuracy of oocyte counts as indicators of numbers of ovulations.

\section{Results}

All control ovaries (from rats in Groups SU, SB and C and from the unoperated sides of rats in Group $U$ ) had moderate to abundant numbers of adrenergic nerve fibres ( $\sim 200-500$ per section) in at least 3 of their 5 representative sections. Of the 19 ovaries from the operated side of rats in Group $\mathrm{U}, 6$ had no nerve fibres in any of their 5 sections; in 7 denervation was nearly complete (1-25 
adrenergic nerve fibres per 5 sections); and the remaining 6 had sparse to moderate numbers of fibres ( $\sim 26-200$ fibres). There were no nerve fibres in any of the sections from 18 of the 28 ovaries from the rats in Group B: 7 of these rats were completely bilaterally denervated.

\section{Ovulation}

The completely denervated ovaries from rats in Groups $U$ and $B$ ovulated normal numbers (mean \pm s.e.m.) of oocytes $(5.8 \pm 1.0$ and $5.2 \pm 1 \cdot 2$, in Groups $U(N=6)$ and $B(N=14)$ respectively) compared with those from Group C (5.6 $\pm 0.4, N=32)$, Group SU (4.4 $\pm 0.6, N=16)$ and Group SB $(4 \cdot 1 \pm 0 \cdot 8, N=16)(P>0 \cdot 05$, unpaired $t$ tests). In addition, the numbers of oocytes ovulated from completely denervated ovaries in rats in Group $U$ were similar to those from the unoperated contralateral ovaries (5.8 \pm 1.0 and $5.0 \pm 1.0$, respectively, $P>0.05$, paired $t$ test).

\section{Agreement of CL and tubal oocyte counts}

In 7 out of 8 rats in Group $U$, and in 2 Group-B rats for which counts were obtained, the number of fresh CL in the ovary and the number of oocytes in the corresponding oviduct were in agreement. In the 8th rat in Group U, there was agreement for the operated side but not for the unoperated side ( $7 \mathrm{CL}, 9$ oocytes).

\section{Oestrous cycles}

Denervation of the ovary did not affect the length of the oestrous cycle in rats in Groups U or B; there was no significant difference from values in the other groups. However, the operative procedure itself did lengthen the cycle since the mean lengths after the operation were greater than those before the operation in all groups. The mean ( \pm s.e.m.) length rose from $4.5 \pm 0 \cdot 2$ days before operation to $4.9 \pm 0.2$ days after operation in Group SU $(P<0.02$, paired $t$ test $)$, from $4.3 \pm 0.2$ to $5.2 \pm 0.2$ days in Group SB $(P<0.01)$, from $4.8 \pm 0.3$ to $5.0 \pm 0.4$ days in Group $U$ (not significant); and from $4 \cdot 3 \pm 0.3$ to $4.7 \pm 0.2$ days in Group B (not significant).

\section{Discussion}

The results clearly indicate that, in rats, ovulation can still occur at a normal rate after complete sympathetic denervation of the ovary. It seems therefore that the mechanism of follicular rupture is independent of any influence from the ovarian sympathetic innervation. These results agree with those from early studies on sympathectomized rats (Sweet \& Thorp, 1929) and rabbits (Brooks, 1935 ) and with those of Weiner, Wright $\&$ Wallach (1975) who found that completely sympathetically denervated rabbit ovaries ovulate normal numbers of oocytes. They also agree with the results from several studies showing that isolated ovaries will ovulate normal numbers of oocytes in response to exogenous gonadotrophins (mice: Fritz, Cho \& Biggers, 1965; rabbits: Lambertson, Greenbaum, Wright \& Wallach, 1976; Janson et al., 1982). In contrast, follicular rupture can be prevented in rabbits and hens by systemic administration of the $\alpha$-adrenoceptor blocker, dibenzyline (Ferrando \& Nalbandov, 1969; Virutamusen et al., 1971). This effect is also produced in hens when dibenzyline is injected directly into the follicle wall (Kao \& Nalbandov, 1972).

It is not clear why a discrepancy exists between the effects of surgical denervation and of pharmacological agents. Surgical denervation is known to induce supersensitivity in smooth muscle (Westfall, 1981). Ojeda, Aguado \& Smith (White) (1983) report that this also occurs in ovarian granulosa cells and we therefore cannot rule out the possibility that low concentrations of circulating catecholamines may bind to the adrenoceptors in the ovary and mimic the actions of the neurotransmitter. However, studies in which drugs are used to block the adrenoceptors exclude this 
possibility since the actions of circulating catecholamines, as well as those of the neurotransmitter, would be prevented. Alternatively, pharmacological agents may have additional non-specific actions on the ovary.

It is not clear why oestrous cycle length was increased by the operative procedure. Barbiturate anaesthesia administered to rats on the afternoon of pro-oestrus will block the ovulatory surge of luteinizing hormone and delay ovulation by $24 \mathrm{~h}$ (van der Schoot, 1978). However, this cannot explain the present finding since all operations in this study were performed on the day of metoestrus.

Since the denervation procedure used in this study also denervates the oviduct (Gibson et al., 1984), it was necessary to determine whether the number of tubal oocytes reliably represented ovulation rate. Although completeness of denervation was checked in all other rats, it could not be checked in this part of our work since the ovaries were treated only by conventional histology in order to count the newly formed corpora lutea. In all but one case, the number of fresh corpora lutea in the ovary agreed with the number of oocytes in the corresponding oviduct. It seems, therefore, that oocyte transport to the oviduct is not dependent on ovarian or oviducal innervation. This also appears to be true for mice since Johns, Chlumecky, Cottle \& Paton (1975) found that the fertility of mice was unchanged after nearly complete denervation of the ovaries and oviducts.

The time course of denervation resulting from the procedure used in this study (Gibson et al., 1984) meant that ovaries were denervated for 2-8 days before ovulation occurred. According to Hirshfield \& Midgley (1978) it takes a small antral follicle in the rat 3-4 days to grow to ovulatory size. Our study therefore indicates that normal numbers of follicles grew through from the small antral stage in the absence of any adrenergic innervation. In conclusion then, it seems that the final stages of follicular growth, as well as the mechanism of follicular rupture, are unaffected by the absence of ovarian sympathetic innervation.

This work was supported by Grant Number D18315184 of the Australian Research Grants Scheme and Grant Number M34/83 of the Monash University Special Research Fund.

\section{References}

Bahr, J., Kao, L. \& Nalbandov, A.V. (1974) The role of catecholamines and nerves in ovulation. Biol. Reprod. 10, 273-290.

Brooks, C. McC. (1935) Studies on the neural basis of ovulation in the rabbit. Am. J. Physiol. 113, 18 Abstr.

Deanesly, R. (1956) Cyclic function in ovarian grafts. $J$. Endocr. 13, 211-220.

de la Torre, J.C. \& Surgeon, J.W. (1976) A methodological approach to rapid and sensitive monoamine histofluorescence using a modified glyoxylic acid technique: The SPG method. Histochemistry 49, 81-93.

Dominguez, R. \& Riboni, L. (1971) Failure of ovulation in autografted ovary of hemispayed rats. Neuroendocrinology 7, 164-170.

Ferrando, G. \& Nalbandov, A.V. (1969) Direct effect on the ovary of the adrenergic blocking drug dibenzyline. Endocrinology 85, 38-42.

Friedman, M.H. (1929) The mechanism of ovulation in the rabbit. I. The demonstration of a humoral mechanism. Am. J. Physiol. 89, 438-442.

Fritz, H.I., Cho, W.K. \& Biggers, J.D. (1965) Ovulation from whole ovaries of mice in organ culture. $J$. Cell Biol. 27, Abstr. 31A.

Gibson, W.R., Roche, P.J., Summers, R.J. \& Wylie, S.N.
(1984) Time course of sympathetic denervation of the rat ovary after freezing its nerve supply. J. Reprod. Fert. 72, 429-433.

Hinsey, J.C. \& Markee, J.E. (1932) A search for neurological mechanisms in ovulation. Proc. Soc. exp. Biol. Med. 30, 136-138.

Hirshfield, A.N. \& Midgley, A.R. (1978) Morphometric analysis of follicular development in the rat. Biol. Reprod. 19, 597-605.

Janson, P.O., Le Maire, W.J., Källfelt, B., Holmes, P.V., Cajander, S., Bjersing, L., Wiqvist, N. \& Ahrén, K. (1982) The study of ovulation in the isolated perfused rabbit ovary. 1. Methodology and pattern of steroidogenesis. Biol. Reprod. 26, 456-465.

Johns, A., Chlumecky, J., Cottle, M.K.W. \& Paton, D.M. (1975) Effects of chemical sympathectomy and adrenergic agonists on the fertility of mice. Contraception 11, 563-570.

Kao, L.W.L. \& Nalbandov, A.V. (1972) The effect of antiadrenergic drugs on ovulation in hens. Endocrinology 90, 1343-1349.

Lambertsen, C.J., Greenbaum, D.F., Wright, K.H. \& Wallach, E.E. (1976) In vitro studies of ovulation in the perfused rabbit ovary. Fert. Steril. 27, 178-187.

Mohsin, S. \& Pennefather, J.N. (1979) The sympathetic 
innervation of the mammalian ovary. A review of pharmacological and histological studies. Clin. exp. Pharm. Physiol. 6, 335-354.

Ojeda, S.R., Aguado, L.I. \& Smith (White), S. (1983) Neuroendocrine mechanisms controlling the onset of female puberty: the rat as a model. Neuroendocrinology 37, 306-313.

Sweet, L.K. \& Thorp, E.G. (1929) The effect of lower abdominal sympathectomy on the oestrous cycle. Am. J. Physiol. 89, 50-53.

van der Schoot, P. (1978) Plasma oestradiol and delayed ovulation after administration of sodium pentobarbitone to pro-oestrous 5 day cyclic rats. $J$. Endocr. 77, 325-332.

Virutamasen, P., Hickok, R.L. \& Wallach, E.E. (1971) Local ovarian effects of catecholamines on human chorionic gonadotropin induced ovulation in the rabbit. Fert. Steril. 22, 235-243.
Walles, B., Owman, Ch., Sjöberg, N.-O., Stefenson, A. \& Wallach, E.E. (1980) Influence of noradrenaline on the process of ovulation. Acta obstet. gynecol. scand., Suppl. 93, 64-65, Abstr. 85.

Weiner, S., Wright, K.H. \& Wallach, E.E. (1975) Lack of effect of ovarian denervation on ovulation and pregnancy in the rabbit. Fert. Steril. 26, 1083-1087.

Westfall, D.P. (1981) Supersensitivity of smooth muscle. In Smooth Muscle: an Assessment of Current Knowledge, pp. 285-309. Eds E. Bülbring, A. F. Brading, A. W. Jones \& T. Tomita. Edward Arnold, London.

Wylie, S.N., Roche, P.J. \& Gibson, W.R. (1983) Ovulation after complete sympathetic denervation of the ovary produced by freezing its nerve supply. Proc. Aust. Soc. Reprod. Biol. 15, Abstr. 7.

Received 31 December 1984 